\title{
Topologimeter and the problem of physical interpretation of topology lattice.
}

\author{
A. A. Grib, R. R. Zapatrin \\ A. A. Friedmann Laboratory For Theoretical Physics \\ Sadovaya, 21, \\ 191023, St-Petersburg, Russia.
}

\begin{abstract}
The collection of all topologies on the set of three points is studied treating the topology as quantum-like observable. It turns out to be possible under the assumption of the asymmetry between the spaces of bra- and ket-vectors. The analogies between the introduced topologimeter and the Stern-Gerlach experiments are outlined.
\end{abstract}

\section{Introduction.}

An important problem in modern theoretical physics is the problem of quantum topology. What is quantum topology? Can one have something like a wave function for topologies? Can one speak about probability of this or that topology, and is it possible to speak about probability calculus for topologies? Are "quantum jumps" between different topologies possible? It is well known that in classical physics one can use classical probability measure, but for molecules, atoms, elementary particles and may be gravity one has probability amplitude description in terms of wave functions. This is connected with the fact that the lattice of properties of quantum system is non distributive (non-Boolean) (Birkhoff and von Neumann, 1936), and one has the formalism of Hilbert space, noncommutative operators, wave function and Heisenberg uncertainty relations. 
One can speak about quantum topology if one deals with Planckean scales for space-time when gravity must be quantised. There is other interesting possibility to speak about quantum topology when according to Leinaas and Myrheim (1991) one gives the interpretation of Pauli principle in terms of nontrivial topology for configuration space of many particle system, so that in EPR experiment, when one goes from symmetrised two particle to the product after measuring local observables one has change of topologies. Here we shall investigate the most simple case of discrete topologies. From (Zapatrin, 1993; Sorkin, 1991) one can see that our analysis can give some insight for continuous case as well.

\section{Topologimeter}

In this paper we shall study the properties of the topology lattice for three points, continuing our investigation in (Grib and Zapatrin, 1992). The striking feature of this lattice and of lattices of topologies for the number of points $n$ greater or equal to 3 , is that they are nondistributive. This makes it impossible to have classical measure on these lattices and leads to some resemblance of them to quantum systems, but without Planck's constant. As in (Grib and Zapatrin, 1992), consider the work of a hypothetical 'topologimeter' an apparatus which can ask and obtain answers on the question: "what is the topology of the three-point set?" We come to the conclusion that due to nondistributivity of the lattice one obtains noncausal "quantum jumps" of topologies similar to situation in Stern-Gerlach experiment when complementary observables are measured. Really, consider the triple $a,(a c),(a b)$ of atomic topologies (the notations are the same as in Grib and Zapatrin, 1992):

$$
a \wedge((a c) \vee(a b)) \quad \text { is not equal to } \quad(a \wedge(a c)) \vee(a \wedge(a b))
$$

Here $(a c) \vee(a b)$ for our topologimeter is also some topology defined following Finkelstein (1963) for the case of quantum logic in the sense that if $(a c)$ is "true" then $((a c) \vee(a b))$ is true, if $(a b)$ is true then $(a c) \vee(a b)$ true.

The Boolean-minded observer comes to the following result using the topologimeter. Imagine he sees $a$ is true. Then, since $a=a \wedge(a c) \vee(a b))$ he will say according to his Boolean distributive logic interpreting $\wedge$ as "and", $\vee$ as "or" that $(a c) \vee(a b)$ is true. Then he must say that either $(a c)$ or $(a b)$ 
is true. However, the atomic topologies $a,(a c),(a b)$ are incompatible since from Hasse diagram we see $a \wedge(a c)=(a c) \wedge(a b)=a \wedge(a b)=0$. The escape from this contradiction is to assume the 'non-causal jump of the topology in different moments of time'. The time here plays the crucial role. If $a$ is true for $t=t_{0}$, then the Boolean-minded observer will say that at some other moment $t=t_{1}$ the topology can non-causally "jump" into some $(a c) \vee(a b)$.

So the situation here resembles that for measuring noncommuting spin operators in the Stern-Gerlach experiment. To make this analysis more close to quantum mechanics and to obtain some "quantum topology" one can try to find something like matrix representation of our lattice of topologies to compare it with usual Pauli matrices for spin. In this paper we show that such representation can be constructed. But differently from quantum mechanics one can not have formulation in terms of one Hilbert space and the wave function there. One must have two spaces and our matrices are operators from one space to the other. There are both commuting and non commuting matrices. All this shows that here we have the example of a new system, different from classical as well as from quantum mechanical cases.

Our matrix representation shows that there is the direct correspondence between noncommutativity of operators and nondistributivity of the lattice.

For nondistributive triples $a,(a c),(a b) ; b,(b c),(a b) ; c,(b c),(a c)$ one has representation in terms of noncommuting "bra operators" (matrices): $a$ doesn't commute with $(a c)$ and $(a b), b$ with $(b c)$ and $(a b)$, same with $c$.

There are distributive triples like $a, b, c$, or $(a b),(a c),(b c)$. That is why comparing the work of our "topologimeter" with Stern-Gerlach experiment one can predict the following. If the topology is fixed as "a" "a" is true) then the prediction that at the next moment if the question will be asked:" is " $(a c)$ 'true?" - the answer can be "yes" — the topology will jump from "a" to " $(a c) "$. The same is true if the question will be about $(a b)$. But if the question will be:" if at $t=t_{0} " a$ " is true, is " $b$ " true at the next moment?" - the answer will be "no". The same prediction will be about "c". The situation here resembles measuring of two noncommuting spin projections for the quantum particle with spin 1. For spin 1 case there are three commuting projectors $S_{x}=1, S_{x}=0, S_{x}=-1$ which do not commute with $S_{y}=1, S_{y}=0, S_{y}=-1$.

Nevertheless our lattice differs from quantum logical lattice of spin 1 particle as we have shown it earlier (Grib and Zapatrin, 1992). It is easy to see from Fig.1 that not only $(b),(c)$ commute with $(a)$ but also the atomic 
(bc), not commuting with $(b)$ and $(c)$.

There is no natural orthogonality in the lattice so if one imposes some orthogonality in the 6 -dimensional space $\mathcal{H}_{V}$ by defining a scalar product and treating mutually commuting idempotents as orthogonal projectors one obtains the contradictory system of equations. So, orthogonal subspaces may not correspond to projectors on any topology represented in the lattice Fig.1. This can be treated as some superpositions of topologies which are not observable by our topologimeter.

The most interesting aspect which makes this system different from usual quantum microparticle is that it is "classical" _ "macroscopic". There is no need for macroscopical apparatus to measure complementary properties of some microparticle. This shows that the reason for "jumps" corresponding to wave packet collapse in quantum mechanics is not connected with any intervention of macroscopical apparatus but is due to interpretation of nondistributive lattice by some consciousness with Boolean logics. Asking question, using Boolean logics, consciousness interprete non-Boolean structure in such a way that it uses time in order to charge "yes-no" values for topologies as it is also done in quantum logic.

But contrary to usual Stern-Gerlach experiment for spin 1 case there is no need for two different complementary topologimeters for measuring noncommuting operators. It is enough to have one topologimeter but one must look for two different moments of time in order to check values of complementary topologies.

The absence of different classical measuring apparatuses for this case leads to absence of necessity to have different von Neumann's measuring Hamiltonian (von Neumann, 1955) for complementary observables. There is no "interaction" between Boolean consciousness and non-Boolean system in this case. This makes the investigation of non-Boolean lattice of topologies comparing it with usual quantum logical system important for our understanding of the deep problem of the role of consciousness in measurment theory in quantum physics either. 


\section{REPRESENTATION OF THE TOPOL- OGY LATTICE $\tau(3)$ BY OPERATORS}

The draft scheme of the construction is the following. We intend to represent the elements of the topology lattice $L=\tau(3)$ by operators in a linear space. Two 6-dimensional spaces $\mathcal{H}_{V}$ and $\mathcal{H}_{\Lambda}$ called bra-space and ket-space, respectively are considered. The basis of $\mathcal{H}_{V}$ is labelled by atoms of $L$, and the basis of $\mathcal{H}_{\Lambda}$ is labelled by coatoms of $L$. Then each element of $L$ has the twofold representation: as subspace of $\mathcal{H}_{V}$ (called bra- representation) or as that of $\mathcal{H}_{\Lambda}$ (ket-representation). The meet operation in $L$ is easily described in terms of the bra- representation as the set-theoretic intersections of appropriate subspaces. The joins in $L$ are associated with the set intersections in the ket- representation. The object of the mathematical treatise exposed below is the construction of joins in terms of $\mathcal{H}_{V}$ and meets in terms of $\mathcal{H}_{\Lambda}$.

\subsection{Bra- and ket- representations}

Let $L=\tau(3)$ be the lattice of all topologies on a set $X=\{a, b, c\}$ of 3 points. The atoms of $L$ are the proper weakest topologies on $X$, each of which associated with the only proper (that is, not equal to $\emptyset$ or $X$ ) open subset of $X=\{a, b, c\}$. Denote by $\mathcal{H}_{V}$ the 6 -dimensional linear space with the basis labelled by these subsets:

$$
\mathcal{H}=\operatorname{span}\left\{\mathbf{e}_{\dashv}, \mathbf{e}_{(\dashv\lfloor)}, \mathbf{e}_{\lfloor}, \mathbf{e}_{(\downarrow\rfloor)}, \mathbf{e}_{\rfloor}, \mathbf{e}_{(\dashv\rfloor)}\right\}
$$

The lattice $L=\tau(3)$ is CAC (complete atomistic coatomistic) lattice (Larson and Andima, 1975), that is why each topology $\tau \in L$ is the join of atomic topologies which are weaker than $\tau$. So, $\tau$ can be unambiguously associated with the subspace $V_{\tau} \subseteq \mathcal{H}_{V}$ spanned on the appropriate basis vectors:

$$
V_{\tau}=\operatorname{span}\left\{\mathbf{e}_{A} \mid \quad A \in \tau\right\}
$$

In other words, for any subset $A \subseteq X, \mathbf{e}_{A} \in V_{\tau}$ if and only if the set $A$ is open in the topology $\tau$.

The ket-representation of $L$ is built likewise. The coatoms of $L$ are associated with the maximal proper topologies on $X=\{a, b, c\}$ each of which is

associated with an ordered pair of elements of $X$ (Zapatrin, 1993a), denoted by $a \rightarrow b, c \rightarrow a$, etc. For example: 


$$
\begin{aligned}
& a \rightarrow b \text { is }\{\emptyset,\{b\},\{c\},\{b, c\},\{a, b\}, X\} \\
& c \rightarrow a \text { is }\{\emptyset,\{a\},\{b\},\{a, b\},\{a, c\}, X\}
\end{aligned}
$$

and so on. Now introduce the 6-dimensional ket-space $\mathcal{H}_{\Lambda}$ with the basis labelled by ordered pairs of $X=\{a, b, c\}$ :

$$
\mathcal{H}_{\Lambda}=\operatorname{span}\left\{\mathbf{f}_{a b}, \mathbf{f}_{b a}, \mathbf{f}_{a c}, \mathbf{f}_{c a}, \mathbf{f}_{b c}, \mathbf{f}_{c b}\right\}
$$

Each topology $\tau \in L$ can now be represented as the subspace $\Lambda_{\tau} \subseteq \mathcal{H}_{\Lambda}$ spanned on the basis vectors $\mathbf{f}_{x y}, x, y \in X$ satisfying the following condition:

$$
\mathbf{f}_{x y} \in \Lambda_{\tau} \quad \Leftrightarrow \quad(\forall A \in \tau \quad x \in A \Rightarrow y \in A)
$$

\section{$2.2 \quad$ Examples}

Six examples of topologies are presented on the Table 2.2.

\begin{tabular}{l||l||l}
\hline Topology $\tau$ & Bra-representation & Ket-representation \\
\hline$a=\{\emptyset,\{a\}, X\}$ & $V_{a}=\operatorname{span}\left\{\mathbf{e}_{a}\right\}$ & $\Lambda_{a}=\operatorname{span}\left\{\mathbf{f}_{b a} \mathbf{f}_{c a} \mathbf{f}_{b c} \mathbf{f}_{c b}\right\}$ \\
(atomic topology) & $\operatorname{dim} V_{a}=1$ & $\operatorname{dim} \Lambda_{a}=4$ \\
\hline$=\{\emptyset,\{a, b\}, X\}$ & $V_{\tau}=\operatorname{span}\left\{\mathbf{e}_{(a b)}\right\}$ & $\Lambda_{\tau}=\operatorname{span}\left\{\mathbf{f}_{b a} \mathbf{f}_{c a} \mathbf{f}_{a b} \mathbf{f}_{c b}\right\}$ \\
(atomic topology) & $\operatorname{dim} V_{(a b)}=1$ & $\operatorname{dim} \Lambda_{(a b)}=4$ \\
\hline$a \vee b=a b(a b)=$ & $V_{\tau}=\operatorname{span}\left\{\mathbf{e}_{a}, \mathbf{e}_{b}, \mathbf{e}_{(a b)}\right\}$ & $\Lambda_{\tau}=\operatorname{span}\left\{\mathbf{f}_{c a} \mathbf{f}_{c b}\right\}$ \\
$=\{\emptyset, a, b,\{a, b\}, X\}$ & $\operatorname{dim} V_{\tau}=3 \neq \operatorname{dim} V_{a}+\operatorname{dim} V_{b}$ & $\operatorname{dim} \Lambda_{\tau}=2$ \\
\hline$a(b c)=\{\emptyset, a,\{b, c\}, X\}$ & $V_{\tau}=\operatorname{span}\left\{\mathbf{e}_{a}, \mathbf{e}_{(b c)}\right\}$ & $\Lambda_{\tau}=\operatorname{span}\left\{\mathbf{f}_{b c} \mathbf{f}_{c b}\right\}$ \\
& $\operatorname{dim} V_{\tau}=2$ & $\operatorname{dim} \Lambda_{\tau}=2$ \\
\hline$(a \rightarrow b)=\{\emptyset, b, c,\{b, c\}$, & $V_{\tau}=\operatorname{span}\left\{\mathbf{e}_{b}, \mathbf{e}_{c}\right.$, & $\Lambda_{\tau}=\operatorname{span}\left\{\mathbf{f}_{a b}\right\}$ \\
$\{a, b\}, X\}$ & $\left.\mathbf{e}_{(a b)}, \mathbf{e}_{(b c)}\right\}$ & \\
(coatomic topology) & $\operatorname{dim} V_{\tau}=4$ & $\operatorname{dim} \Lambda_{\tau}=4$ \\
\hline
\end{tabular}

Table 1: Some examples of topologies 


\subsection{Transition between the representations}

Let $\tau \in L$ is represented by the bra-subspace $V_{\tau} \subseteq \mathcal{H}_{V}$. Denote by $P_{\tau}$ the projector onto $V_{\tau}$. Let us construct the algorithm which builds $\Lambda_{\tau}$ by given $V_{\tau}$, and vice versa. To proceed it, first introduce the sandwich operator $S: \mathcal{H}_{V} \rightarrow \mathcal{H}_{\Lambda}$ in matrix form as:

$$
S_{\lambda v}= \begin{cases}0 & , v \leq \lambda \text { (i.e. the atom } v \text { is below the coatom } \lambda \text { ) } \\ 1 & , \text { otherwise }\end{cases}
$$

Its transposed $S^{T}$ will be the operator $S^{T}: \mathcal{H}_{\Lambda} \rightarrow \mathcal{H}_{V}$. Consider the product $S P_{\tau}: \mathcal{H}_{V} \rightarrow \mathcal{H}_{\Lambda}$. In the space $\mathcal{H}_{\Lambda}$, denote by $\Pi_{\tau}$ the projector onto the subspace $\Lambda_{\tau}$. In particular, denote the projectors onto the basis vectors $\mathbf{e}_{x y}, x, y \in\{a, b, c\}$ by $\pi_{x y}$.

Lemma 1 Let $\tau \in L$ be a topology on $X=\{a, b, c\}, V_{\tau}$ be its bra-representation, and $P_{\tau}$ be the projector in $\mathcal{H}_{V}$ associated with $V_{\tau}$. Then the projector $\Pi_{\tau}$ in $\mathcal{H}_{\Lambda}$ associated with $\Lambda_{\tau}$ is:

$$
\Pi_{\tau}=\Sigma\left\{\pi_{x y} \mid \quad \pi_{x y} S P_{\tau}=0\right\}
$$

Proof. $\pi_{x y}$ is included in the sum (4) if and only if $S(x y, v)=0$ for any atomic topology $v$ which is weaker then $\tau$. Due to (R.3) that means that $\pi_{x y} \leq \Pi_{\tau}$ iff

$$
\forall v v \leq \tau \Leftrightarrow v \leq \lambda
$$

Since the lattice $L$ is coatomistic, $\tau=\wedge\{\lambda \mid \tau \leq \lambda\}$, thus (3) implies (4).

The following "transposed" Lemma is proved likewise. Denote by $p_{A}$ the projector onto the vector $\mathbf{e}_{A}$ in $\mathcal{H}_{V}$.

Lemma 2 The transition from the ket- to bra-representation is described as follows:

$$
P_{\tau}=\sum\left\{p_{A} \mid p_{A} S^{T} \Pi_{\tau}=0\right\}
$$




\subsection{Lattice joins in bra-representation}

Let $\sigma, \tau \in L$, and let their bra-representations be $V_{\sigma}, V_{\tau} \subseteq \mathcal{H}_{V}$, associated with the projectors $P_{\sigma}, P_{\tau}$, respectively. To build the projector $P_{\sigma \vee \tau}$, perform consecutively the transition procedures described in the Lemmas ??. First form the ket-representation $\Lambda_{\sigma \vee \tau}$ associated with the projector (丑):

$$
\Pi_{\sigma \vee \tau}=\sum\left\{\pi_{x y} \mid \pi_{x y} S\left(P_{\sigma}+P_{\tau}\right)=0\right\}
$$

and then go backwards to $\mathcal{H}_{V}$. (R.6) is really the projector associated with the join since it is the meet of all upper bounds for both $\sigma$ and $\tau$ (Zapatrin, 1994).

\subsection{An example}

Let us explicitly build the projector associated with the join of two atomic topologies $a$ and $b$.

$$
\begin{aligned}
\mathbf{e}_{a}=\left[\begin{array}{l}
1 \\
0 \\
0 \\
0 \\
0 \\
0
\end{array}\right] & \Rightarrow P_{a}=\left[\begin{array}{llllll}
1 & 0 & 0 & 0 & 0 & 0 \\
0 & 0 & 0 & 0 & 0 & 0 \\
0 & 0 & 0 & 0 & 0 & 0 \\
0 & 0 & 0 & 0 & 0 & 0 \\
0 & 0 & 0 & 0 & 0 & 0 \\
0 & 0 & 0 & 0 & 0 & 0
\end{array}\right] \\
\mathbf{e}_{b}=\left[\begin{array}{l}
0 \\
0 \\
1 \\
0 \\
0 \\
0
\end{array}\right] & \Rightarrow P_{b}=\left[\begin{array}{llllll}
0 & 0 & 0 & 0 & 0 & 0 \\
0 & 0 & 0 & 0 & 0 & 0 \\
0 & 0 & 1 & 0 & 0 & 0 \\
0 & 0 & 0 & 0 & 0 & 0 \\
0 & 0 & 0 & 0 & 0 & 0 \\
0 & 0 & 0 & 0 & 0 & 0
\end{array}\right]
\end{aligned}
$$

Set up the following order of basis vectors in $\mathcal{H}_{V}: \mathbf{e}_{a}, \mathbf{e}_{a b}, \mathbf{e}_{b}, \mathbf{e}_{b c}, \mathbf{e}_{c}, \mathbf{e}_{a c}$, and of those in $\mathcal{H}_{\Lambda}: \mathbf{f}_{c a}, \mathbf{f}_{b a}, \mathbf{f}_{b c}, \mathbf{f}_{a c}, \mathbf{f}_{a b}, \mathbf{f}_{c b}$, then the matrix of the sandwich 
operator is:

$$
S=\left[\begin{array}{llllll}
1 & 1 & 0 & 0 & 0 & 0 \\
0 & 1 & 1 & 0 & 0 & 0 \\
0 & 0 & 1 & 1 & 0 & 0 \\
0 & 0 & 0 & 1 & 1 & 0 \\
0 & 0 & 0 & 0 & 1 & 1 \\
1 & 0 & 0 & 0 & 0 & 1
\end{array}\right]
$$

Then

$$
P_{a}+P_{b}=\left[\begin{array}{cccccc}
1 & 0 & 0 & 0 & 0 & 0 \\
0 & 0 & 0 & 0 & 0 & 0 \\
0 & 0 & 1 & 0 & 0 & 0 \\
0 & 0 & 0 & 0 & 0 & 0 \\
0 & 0 & 0 & 0 & 0 & 0 \\
0 & 0 & 0 & 0 & 0 & 0
\end{array}\right] \quad \text {, hence } \quad S\left(P_{a}+P_{b}\right)=\left[\begin{array}{cccccc}
1 & 0 & 0 & 0 & 0 & 0 \\
0 & 0 & 1 & 0 & 0 & 0 \\
0 & 0 & 1 & 0 & 0 & 0 \\
0 & 0 & 0 & 0 & 0 & 0 \\
0 & 0 & 0 & 0 & 0 & 0 \\
1 & 0 & 0 & 0 & 0 & 0
\end{array}\right]
$$

Therefore the only $\pi_{x y}$ satisfying 5 are the projectors onto the following vectors

$$
\begin{aligned}
\mathbf{f}_{b c} & =(0,0,1,0,0,0) \\
\mathbf{f}_{c a} & =(1,0,0,0,0,0)
\end{aligned}
$$

hence

$$
\Pi_{a \vee b}=\pi_{b c}+\pi_{c a}=\left[\begin{array}{cccccc}
1 & 0 & 0 & 0 & 0 & 0 \\
0 & 0 & 0 & 0 & 0 & 0 \\
0 & 0 & 1 & 0 & 0 & 0 \\
0 & 0 & 0 & 0 & 0 & 0 \\
0 & 0 & 0 & 0 & 0 & 0 \\
0 & 0 & 0 & 0 & 0 & 0
\end{array}\right]
$$

Then, it follows from the Lemma 2 that

$$
S^{T} \Pi_{a \vee b}=\left[\begin{array}{cccccc}
1 & 0 & 0 & 0 & 0 & 1 \\
1 & 1 & 0 & 0 & 0 & 0 \\
0 & 1 & 1 & 0 & 0 & 0 \\
0 & 0 & 1 & 1 & 0 & 0 \\
0 & 0 & 0 & 1 & 1 & 0 \\
0 & 0 & 0 & 0 & 1 & 1
\end{array}\right] \circ\left[\begin{array}{llllll}
1 & 0 & 0 & 0 & 0 & 0 \\
0 & 0 & 0 & 0 & 0 & 0 \\
0 & 0 & 1 & 0 & 0 & 0 \\
0 & 0 & 0 & 0 & 0 & 0 \\
0 & 0 & 0 & 0 & 0 & 0 \\
0 & 0 & 0 & 0 & 0 & 0
\end{array}\right]=\left[\begin{array}{llllll}
1 & 0 & 0 & 0 & 0 & 0 \\
1 & 0 & 0 & 0 & 0 & 0 \\
0 & 0 & 1 & 0 & 0 & 0 \\
0 & 0 & 1 & 0 & 0 & 0 \\
0 & 0 & 0 & 0 & 0 & 0 \\
0 & 0 & 0 & 0 & 0 & 0
\end{array}\right]
$$

The $p_{A}$ 's satisfying (5) are the projectors onto the vectors: 


$$
\mathbf{e}_{a}=\left[\begin{array}{c}
1 \\
0 \\
0 \\
0 \\
0 \\
0
\end{array}\right] \quad \mathbf{e}_{(a b)}=\left[\begin{array}{c}
0 \\
1 \\
0 \\
0 \\
0 \\
0
\end{array}\right] \quad \mathbf{e}_{b}=\left[\begin{array}{l}
0 \\
0 \\
1 \\
0 \\
0 \\
0
\end{array}\right]
$$

Hence

$$
\Pi_{a \vee b}=\pi_{a}+\pi_{a b}+\pi_{b}=\left[\begin{array}{cccccc}
1 & 0 & 0 & 0 & 0 & 0 \\
0 & 1 & 0 & 0 & 0 & 0 \\
0 & 0 & 1 & 0 & 0 & 0 \\
0 & 0 & 0 & 0 & 0 & 0 \\
0 & 0 & 0 & 0 & 0 & 0 \\
0 & 0 & 0 & 0 & 0 & 0
\end{array}\right] \neq \pi_{a}+\pi_{b}
$$

The other joins in $\mathcal{H}_{V}$ are built in the same way.

\section{Commutation relations}

At first sight, the proposed representation of property lattices seems inconsistent with quantum mechanical intuition since the operators associated with the atoms of property lattice all commute. To reason about the commutativity of observables we must somehow take into account both bra- and ket-representation at once.

For instance, consider the pair of operators $P_{a}$ and $P_{(a b)}$. They act from the bra-space to the ket-space, therefore it makes no sense to speak about they commutation since they can not be multiplied. To speak about commutation relations, we have to render them to the same space. Note that we already have the operator doing it, namely, the sandwich matrix $S$ (6), and the operators $S P_{a}$ and $S P_{(a b)}$ will already act in the same space having the form:

$$
S P_{a}=\left[\begin{array}{cccccc}
1 & 0 & 0 & 0 & 0 & 0 \\
0 & 0 & 0 & 0 & 0 & 0 \\
0 & 0 & 0 & 0 & 0 & 0 \\
0 & 0 & 0 & 0 & 0 & 0 \\
0 & 0 & 0 & 0 & 0 & 0 \\
1 & 0 & 0 & 0 & 0 & 0
\end{array}\right] \quad ; \quad S P_{(a b)}=\left[\begin{array}{cccccc}
0 & 1 & 0 & 0 & 0 & 0 \\
0 & 1 & 0 & 0 & 0 & 0 \\
0 & 0 & 0 & 0 & 0 & 0 \\
0 & 0 & 0 & 0 & 0 & 0 \\
0 & 0 & 0 & 0 & 0 & 0 \\
0 & 0 & 0 & 0 & 0 & 0
\end{array}\right]
$$


It can be checked directly that both they are idempotents: $\left(S P_{a}\right)^{2}=S P_{a}$ and $\left(S P_{(a b)}\right)^{2}=S P_{(a b)}$. We could try to make them projectors (that is, self adjoint operators) by introducing a scalar product in the bra-space in, say, usual Euclidean way. Although, unlike the quantum mechanical situation, they will not be self adjoint: $\left(S P_{a}\right)^{T} \neq S P_{a},\left(S P_{(a b)}\right)^{T}=S P_{(a b)}$.

We have to introduce commutation relation in such a way that they could grasp the entire structure of the property lattice. When we try to use the introduced above projectors $P_{a}, P_{b}$ etc., we immediately see that they all commute which contradicts to the violation of distributivity (1). The idea we put forth is the following. Since all the operators associated with the elements of the lattice act from $\mathcal{H}_{V}$ to $\mathcal{H}_{\Lambda}$, we can render them into one space, namely $\mathcal{H}_{V}$, by multiplying all of them by the matrix $S$ from the right side. Then define the new product $\circ$ of operators in $\mathcal{H}_{V}$

$$
A \circ B:=A S B S
$$

and calculate all commutators $\left[P_{u}, P_{v}\right]$ for all $u, v \in V$.

Lemma 3 For any $u, v \in V$

$$
\left[P_{u}, P_{v}\right]=S_{u v}\left(P_{u}-P_{v}\right)=\left\{\begin{array}{lll}
= & 0 & \text { if } S_{u v}=0 \\
\neq & 0 & \text { otherwise }
\end{array}\right.
$$

Proof is obtained from direct checking by multiplication of appropriate matrices.

The results of the calculation are shown on the Figure ?? where the vertices associated with commuting projectors are linked by lines of the diagram, and the pairs of vertices which are not connected by a line, are associated with non-commuting projectors. Now the correspondence between commutativity of projectors and distributivity of the elements of the lattice is gathered. For instance, the atomic topologies $a, b, c$ form the distributive triple, and the appropriate projectors pairwise commute.

\section{Concluding remarks}

So, we see that the matrix representation of topology lattice for three points is possible if one uses two spaces, called "bra"- and "ket"-spaces. Contrary 
to usual quantum mechanics, it is impossible to identify these spaces and to have usual wave function formulation in terms of vectors in one space. It is onle the special case of the 3-point set on which all possible topologies are studied that makes it possible to reduce the construction to one space, since the bra- and ket-spaces are isomorhic only when $n=3$.

Noncommutativity of some of these matrices can lead to complementarity (as in the case of Stern-Gerlach experiment) and to quantum jumps for topologimeter. Nevertheless one must stress that the example of topologimeter for the lattice of topologies for three points is the example of totally new system, different from both classical and quantum systems. From this one

comes to conclusion that quantum topology can not be thought of as some usual quantum system described by the wave functions as vectors in one Hilbert space, but a new formalism for which a new interpretation is needed.

\section{Acknowledgments}

One of the authors (A.A.G.) is thankful to CNPq of Brasil for financial support when completing this work.

\section{References}

Birkhoff, G, and J. von Neumann, (1936), The logic of quantum mechanics, Annals of Mathematics, 37, 923

Finkelstein, D., (1963), Transactions of the New York Academy of Science, 25, 621

Grib, A.A., and R.R.Zapatrin, (1992), Topology Lattice As Quantum Logic, International Journal of Theoretical Physics, 31, 1093

Larson R.F., and S.Andima, (1975), The lattice of topologies: a survey, Rocky Mountains Journal of Mathematics, 5, 177

Leinaas, J., and R. Myrheim, (1991), Quantum theories for identical particles, International Journal of Modern Physics, B5, 2573

von Neumann, J., (1955), Mathematical Foundations of Quantum Mechanics, Princeton, New Jersey 
Sorkin, R., (1991), Finitary Substitutes For Continuous Topology, International Journal of Theoretical Physics, 30, 930

Zapatrin, R.R., (1993), Pre-Regge Calculus: Topology Via Logic, International Journal of Theoretical Physics, 32, 779

Zapatrin, R.R., (1994), Quantum Logic Without Negation, Helvetica Physica Acta, 67, 188

D'Espagnat, B., (1976), Conceptual foundations of quantum mechanics, Benjamin , New York 


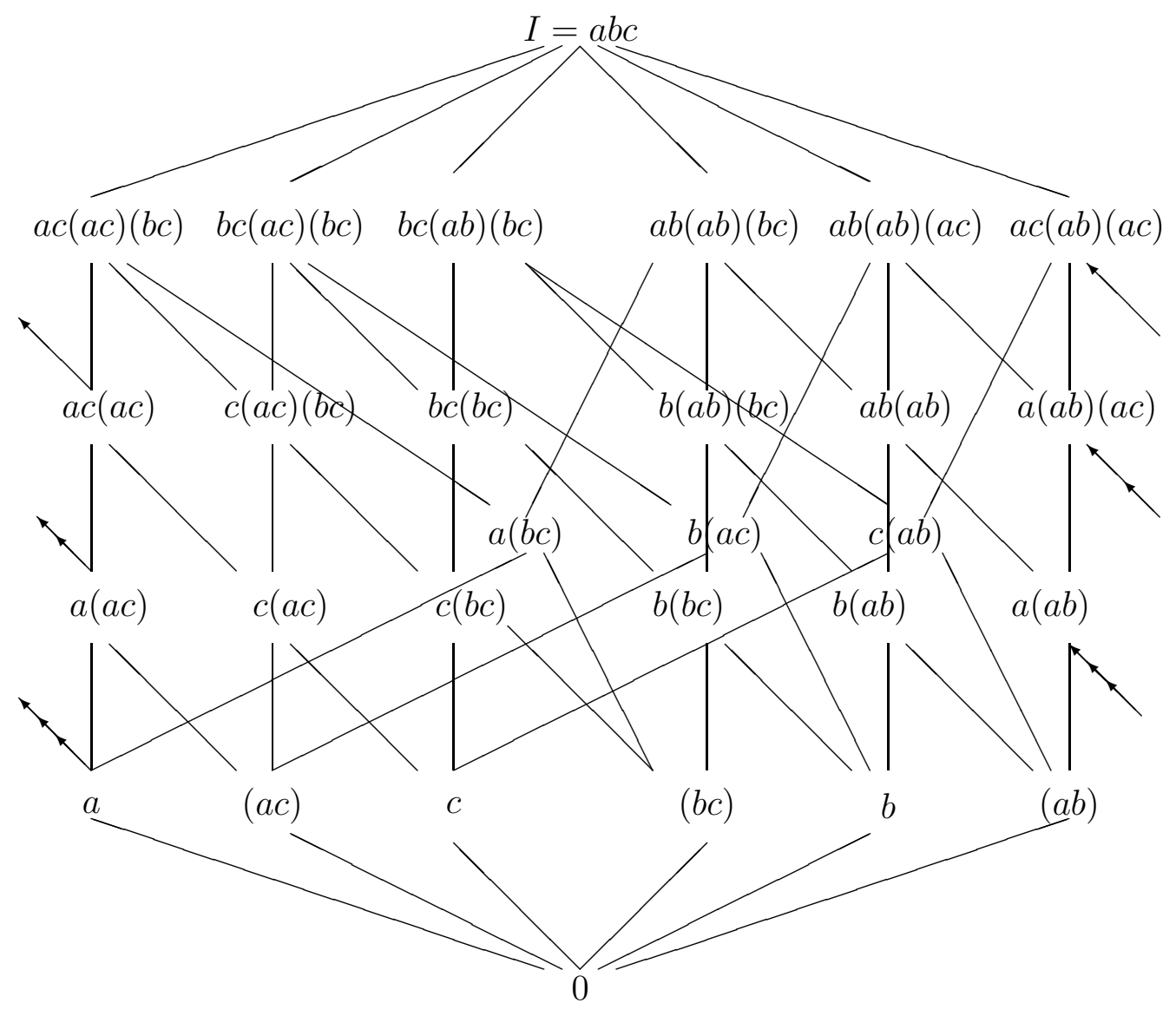

Figure 1: The lattice $\tau(3)$. 


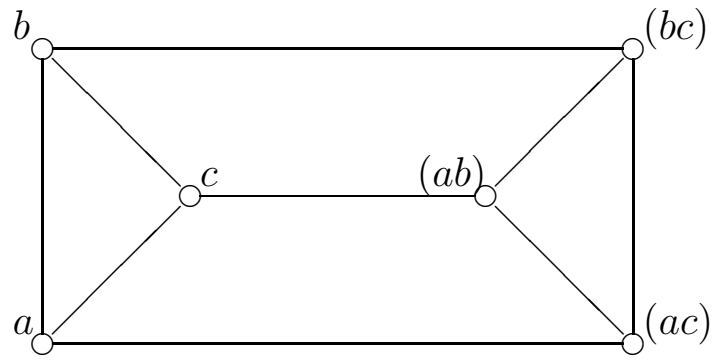

Figure 2: Commutativity of atomic projectors 